\title{
Una Revisión sobre Materiales para Almacenamiento de Energía Solar Térmica \\ Debrayan Bravo Hidalgo
}

${ }^{1}$ Universidad San Francisco de Quito (USFQ), Quito, Ecuador

Correspondence: correspondence email: dbrayanbh@gmail.com

Recibido: 03/10/2017. Modificado: 23/01/2018. Aceptado: 30/01/2018.

\begin{abstract}
Context: The energy and environmental panorama that our societies confront nowadays, demand for renewable, clean and abundant sources of energy, not reliant on fossil fuels and detached from the geopolitical pressures the latter represent. In this scenario solar thermal energy arises as a viable and functional option. The main disadvantage of this emerging source of energy lies in the intermittent availability of solar radiation. Because of this difficulty, efficient processes for the storage of thermal solar energy becomes a highly relevant area of research. The aim of this paper is to present a survey on this topic.
\end{abstract}

Method: A bibliographic review was carried out using the Scopus catalog. The search criteria for this purpose was defined using the following terms: solar+thermal+energy+storage+materials. This search pattern was applied to the title, abstracts and keywords of the contributions. Using the bibliometric tools of the citation database, the most cited documents were selected and the survey was developed.

Results: A growing interest in the scientific community regarding this energy practice is evident starting from 2010. Characteristics, advances and trends in systems that use thermal energy storage materials are presented for sensible and latent heat, materials compound changeover phase, and finally thermochemical thermal storage materials.

Conclusions: Improving the thermal conductivity of thermal storage materials is an important trend in current re-search. On the other hand, profitable practices for micro-encapsulated phase change materials and composite materials are analyzed. The optimization of thermo-physical properties as the melting point of thermal storage materials is explored with techniques such as eutectic mixtures and hydrocarbon chain length. Although the thermochemical materials are still in laboratory stage, they have a great potential as thermal storage materials in the future, given their large energy storage capacity per unit volume.

Keywords: Solar thermal energy, thermal energy storage, phase change materials, bibliographic survey Language: Spanish 


\section{Resumen}

Contexto: El panorama energético y medioambiental que hoy día enfrenta la sociedad, demanda el empleo de fuentes renovables de energía, abundantes, limpias y apartadas de las tensiones geopolíticas asociadas a los combustibles fósiles. En este escenario, la energía solar térmica se presenta como una opción viable y funcional; sin embargo, su desventaja radica en el carácter intermitente de la radiación solar, de allí que los procesos de almacenamiento de energía solar térmica cobren especial relevancia para solucionarlo. El propósito de este artículo es presentar una revisión bibliográfica sobre este tema de interés.

Método: Se realizó una revisión bibliográfica en el catálogo Scopus, utilizando como criterio de búsqueda la frase "solar thermal energy storage materials". Este patrón de búsqueda se aplicó al título, los resúmenes y las palabras claves de los artículos consultados. Con las herramientas de análisis bibliométrico de esta base de datos se seleccionaron los documentos más referenciados, con los cuales se procedió a realizar la revisión.

Resultados: Se destaca un creciente interés en la comunidad científica por esta práctica energética a partir de 2010. Se presentan las características, los avances y tendencias en los sistemas que emplean materiales de almacenamiento de energía térmica por calor sensible y por calor latente, materiales de cambio de fase compuestos y, por último, los materiales termoquímicos de almacenamiento térmico.

Conclusiones: La mejora de la conductividad térmica de los materiales de almacenamiento térmico es un área importante en las actuales investigaciones. Por otra parte, se analizan prácticas rentables para materiales de cambio de fase microencapsulado y materiales compuestos. La optimización de las propiedades termofísicas como el punto de fusión de los materiales de almacenamiento térmico, se exploran con técnicas como las mezclas eutécticas y la longitud de la cadena de hidrocarburos. Los materiales termoquímicos se encuentran en fase de laboratorio, estos tendrán un gran potencial como materiales de acumulación térmica en el futuro dado su gran capacidad de almacenamiento de energía por unidad de volumen.

Palabras clave: Acumulación térmica, energía solar térmica, materiales de cambio de fase, revisión bibliográfica.

Idioma: Español

\section{Introducción}

Aplicaciones como el calentamiento o refrigeración de espacios interiores de edificaciones requieren del almacenamiento de energía térmica (AET) a bajas temperaturas, es decir, por debajo de $50{ }^{\circ} \mathrm{C}$, mientras que aplicaciones como la generación de energía eléctrica requieren sistemas AET de altas temperaturas mayores a los $175^{\circ} \mathrm{C}$ [1], [2]. Las prestaciones de los sistemas AET dependen de las propiedades de los materiales seleccionados para este propósito [3]-[5]. Las propiedades termofísicas de los materiales de almacenamiento de energía térmica deben considerarse de acuerdo con los requerimientos propios de cada campo de aplicación [6]-[8]. Por ello, se deben tener presentes las características mencionadas a continuación.

- Calor latente de fusión: los materiales de cambio de fase deben tener un calor latente de fusión muy alto. El calor latente elevado de la fusión mejora la densidad de almacenamiento del sistema.

- Calor específico (Cp): los materiales sensibles de almacenamiento de calor deben tener un calor específico alto. El alto calor específico mejora la densidad de almacenamiento de energía del sistema. 
- Punto de fusión: los materiales de cambio de fase deben tener un punto de fusión cercano al rango de temperatura de funcionamiento requerido del sistema AET.

- Densidad: la alta densidad mejora la densidad de almacenamiento y reduce el volumen del sistema AET.

- Conductividad térmica: la alta conductividad térmica aumenta la carga térmica y la tasa de descarga que se desea.

- Costo y disponibilidad: el precio más barato del material de almacenamiento reduce los costos de capital y operacionales.

- Súper enfriamiento: para materiales de cambio de fase, durante el proceso de congelación el superenfriamiento debe ser mínimo.

- Estabilidad térmica: no deben descomponerse a altas temperaturas. Esto da un rango de temperatura de operación más amplio. Las propiedades del material deben ser estables incluso después de prolongados ciclos térmicos de calentamiento y enfriamiento.

- Estabilidad química: alta estabilidad química de los materiales de almacenamiento aumenta la vida del sistema de acumulación térmica.

- Cambio de volumen: para los materiales de cambio de fase, el cambio de volumen durante el proceso de cambio de fase debe ser mínimo. Estos materiales deben poseer un bajo coeficiente de expansión térmica. Grandes cambios en el volumen aumentan el tamaño requerido del contenedor.

- Inflamabilidad: deben ser no inflamables y no explosivos.

- Presión de vapor: deben tener baja presión de vapor en el rango de temperatura operativa. En caso contrario se complejiza y encarece la instalación.

- No tóxico: no debe ser perjudicial para la salud de los operadores y el medio ambiente.

- No corrosivo: los materiales de almacenamiento de energía térmica corrosiva reducen drásticamente la vida útil de la instalación de almacenamiento de energía, debido a la corrosión de los contenedores.

Las instalaciones de acumulación térmica permiten disponer de energía en ausencia de luz solar, este hecho atenúa la dificultad que representa la intermitencia del astro rey en el mundo; la tecnología de acumulación térmica también ayuda a suavizar las fluctuaciones en la demanda de energía durante diferentes períodos del día. Actualmente, una de las corrientes investigativas en esta temática son los proyectos enfocados a la optimización de las característica química y física de los materiales de almacenamiento térmico, toda vez que el éxito de cualquier tecnología de acumulación termoenergética depende en gran medida de los materiales seleccionados con este propósito [3], [9]. Esta contribución presenta una análisis crítico, sistemático y exhaustivo, mediante una revisión bibliográfica de los materiales empleados en los distintos procesos de acumulación térmica que operan con plantas de captación de energía solar, detallando la calificación y propiedades físico-químicas de estos materiales. Dado el gran interés que la comunidad científica ha despertado 
por esta práctica energética, se destacan en este trabajo los avances y tendencias del empleo de los materiales de almacenamiento de energía térmica.

\section{Materiales y métodos}

Scopus es una base de datos bibliográfica y documental que contiene artículos, libros, conferencias, sus resúmenes y citas, agrupados estos en revistas científicas de reconocimiento internacional en las más diversas áreas del saber. Este catálogo académico investigativo tiene en su haber alrededor de 18000 títulos de más de 5000 editores internacionales, incluyendo la cobertura de 16500 revistas revisadas por pares [10]. A través de esta plataforma científica online, se realizó una revisión bibliográfica la cual se implementó estableciendo como criterio de búsqueda la frase "solar thermal energy storage materials"; este patrón de búsqueda se orientó al título, los resúmenes y las palabras clave de las contribuciones. De esta forma, se detectaron 2820 documentos y 72328 patentes registradas. Con las herramientas de análisis bibliométrico que brinda esta plataforma se seleccionaron los documentos más referenciados entre las investigaciones concernientes a los estudios sobre materiales para el almacenamiento de energía solar térmica. Dichas herramientas permitieron, además, detectar el comportamiento de la productividad científica y de patentes en los últimos dieciséis años, así como el desempeño bibliométrico de las revistas que más socializan resultados investigativos vinculados con la acumulación térmica. Los gráficos contenidos en este documento se generaron con el software MATLAB 2017a.

\subsection{Limitaciones de la investigación}

La revisión abarca solo resultados publicados en idioma ingles y contenidos en el directorio Scopus. Se consideran solo contribuciones del 2004 en adelante, exceptuando una única publicación de 1998, por contar con un índice Hirsch $(\mathrm{H})$ de 550. El 67.6\% de las referencias bibliográficas contenidas en este informe se concentran en los últimos tres años.

\section{Resultados}

\subsection{Sinopsis bibliométrica de la temática "Materiales de almacenamiento de energía solar térmica” en el catálogo Scopus}

Bajo los criterios de búsqueda declarados en la sección materiales y métodos, y empleando las herramientas de investigación y análisis de estadística bibliométrica que este catálogo ofreces a sus suscriptores, se generó empleando MATLAB la Figura 1, esta destaca el comportamiento tanto de las contribuciones científicas en esta área temática, como las patentes registradas en el mismo periodo de tiempo. Se puede observar que a partir del 2010 la comunidad científica internacional marca un notable incremento en las investigaciones dirigidas a las tecnologías de acumulación térmica y sus prácticas.

Las revistas que más socializan esta temática son, Solar Energy, Energy Procedia, Applied Energy, Applied Thermal Engineering y Solor Energy Materials and Solar Cells, según Scopus. La Figura 2 


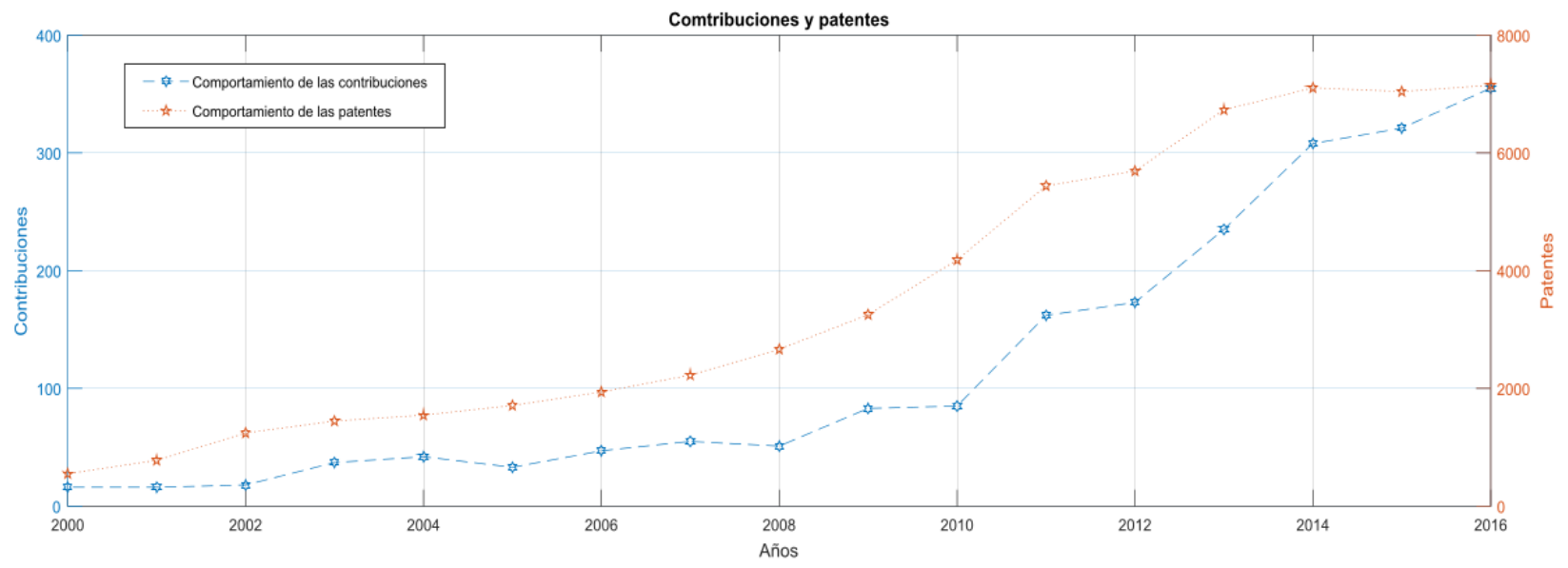

Figura 1. Comportamiento del número de contribuciones y patentes registradas dentro del directorio académico Scopus, bajo el criterio de búsqueda anteriormente mencionado. TITLE-ABS-KEY (solar AND thermal AND energy AND storage AND materials )

muestra el comportamiento del patrón de contribuciones por cada una de estas revistas del 2000 al 2016, también se muestra el patrón de citas bibliográficas por cada una de estas prestigiosas editoras científicas.
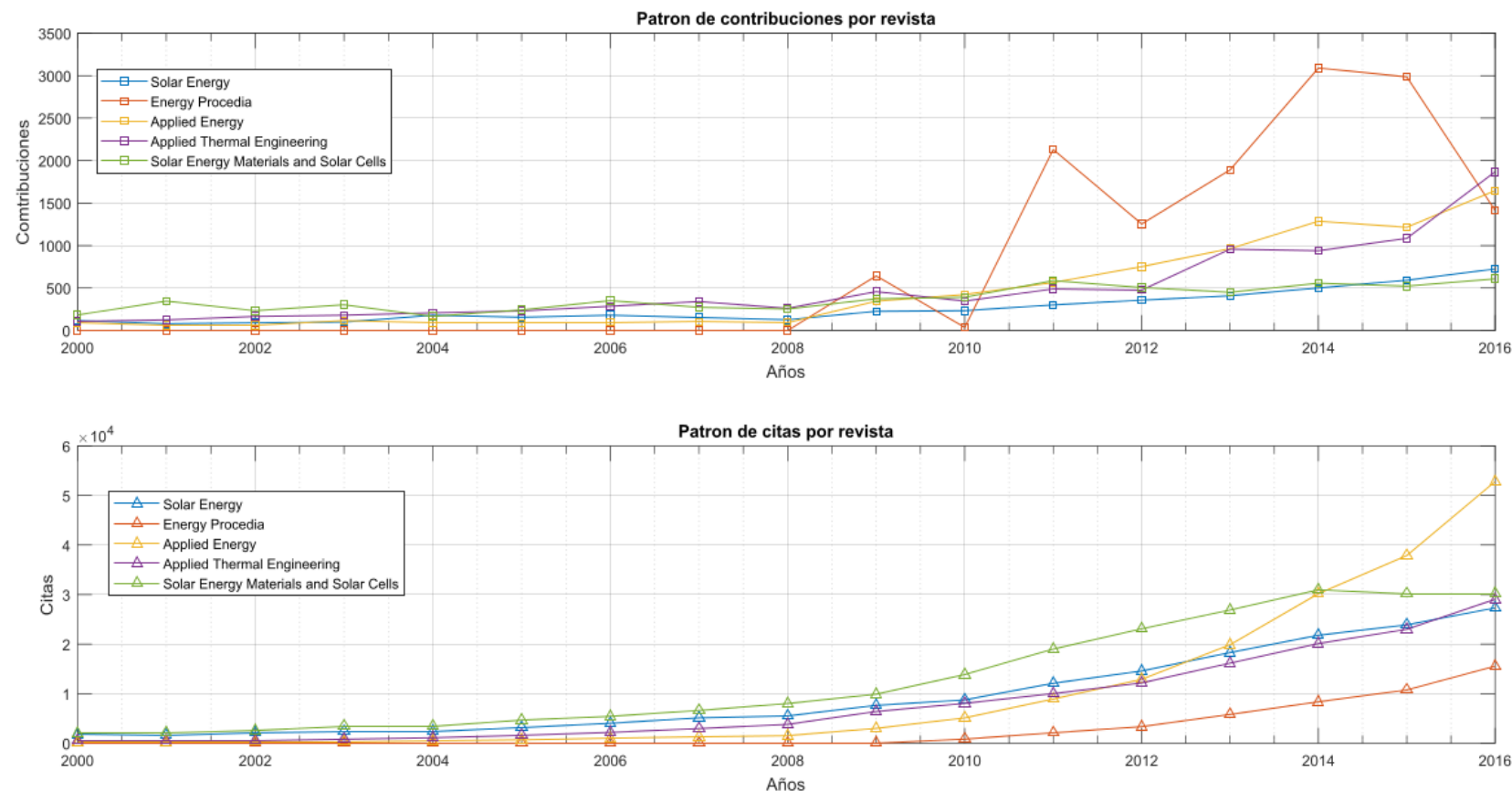

Figura 2. Análisis bibliométrico sobre la temática tratada, dentro de las revistas que más representatividad ofrecen a esta línea de trabajo dentro de Scopus. TITLE-ABS-KEY (solar AND thermal AND energy AND storage AND materials)

Tanto la Figura 1 como la Figura 2 evidencian el notable interés de investigadores e ingenieros en la alternativa de acumulación de energía solar térmica, evidencias que justifican y fundamentan la pertinencia de la presente contribución, ya que esta describe y analiza las distintas prácticas de acumulación térmica a partir de los materiales que se emplean con este propósito. 


\subsection{Materiales de almacenamiento de energía térmica por calor sensible}

Los materiales de almacenamiento de energía térmica por calor sensibles acumulan energía por su capacidad calorífica específica $(\mathrm{Cp})$. La energía térmica almacenada por calor sensible puede expresarse como:

$$
Q=m * C p * \Delta T
$$

Donde

$m$ : es la masa $(\mathrm{kg})$.

$C p$ : es la capacidad calorífica específica $(k J / k g * K)$.

$\Delta T$ : es la diferencia de la temperatura durante el proceso de carga o descarga, es decir durante la absorción o cesión de energía térmica $(\mathrm{K})$.

A continuación, se detallan algunos de los materiales de almacenamiento por calor sensibles más comunes en los sistemas de la acumulación de energía solar térmica.

\subsubsection{Medio de almacenamiento líquido}

La ventaja de un medio de almacenamiento en estado líquido es que se puede hacer circular fácilmente y por lo tanto puede transportar calor con eficiencia. Los sistemas en los que el medio de almacenamiento es un fluido se denominan sistemas activos; además, debido a la diferencia de densidad causada por el gradiente térmico del fluido, se crea el termoclima que puede ser utilizado a favor del proceso [11], [12]. El líquido caliente sube y el líquido frío se desplaza hacia abajo separados por el termoclima. A continuación, se describen algunos de estos fluidos de trasferencia de calor (FTC):

\subsubsection{Agua}

El agua es uno de los mejores medios de almacenamiento para aplicaciones a baja temperatura. $\mathrm{Su}$ rango de temperatura de funcionamiento está entre $8-90{ }^{\circ} \mathrm{C}$ [13], [14]. Sus ventajas son el elevado calor especifico, la no toxicidad y la gran disponibilidad, pero tiene algunos inconvenientes como la alta presión de vapor y la corrosividad. El agua se utiliza para la calefacción y refrigeración de espacios interiores, y como servicio de agua caliente sanitaria. El agua salada en los estanques solares se utiliza para recoger gran cantidad de energía solar térmica a bajas temperaturas $\left(50-95^{\circ} \mathrm{C}\right)$. El calor llega a la capa densa en la parte inferior del estanque debido a la sal disuelta que impide la convección natural. Se usan sales como $\mathrm{NaCl}$ y $\mathrm{MgCl}_{2}$.

Los tanques de almacenamiento de agua están hechos de una amplia variedad de materiales como acero, aluminio, hormigón armado y fibra de vidrio, están aislados con lana de vidrio, lana mineral o poliuretano. Los tamaños de los tanques utilizados varían de unos pocos cientos de litros a miles de metros cúbicos, los grandes tanques de agua requieren el desarrollo de tecnologías capaces de garantizar la estanqueidad al agua, de minimizar las pérdidas de calor causadas por el vapor a través de las paredes y de optimizar la estratificación dentro del tanque, para preservar el rendimiento térmico y la vida útil de la planta solar. El agua también puede utilizarse para fines de almacenamiento de energía térmica a gran escala en acuíferos subterráneos donde el agua podría 
encontrarse mezclada con grava, un sistema de este tipo es muy rentable ya que se puede evitar costos de construcción de tanques de agua [15], [16].

\subsubsection{Aceite mineral}

El aceite mineral se utiliza como FTC en plantas de captación de energía solar térmica (PCEST) para colectar el calor en el receptor y luego transporta el calor al generador de vapor para impulsar las turbinas, este es su propósito; puede usarse también para almacenar energía térmica en tanques de almacenamiento altamente aislados y disponer de energía durante las noches. Cuando el FTC también se convierte en material de almacenamiento de energía, se les denomina a las instalaciones de sistema directo, puesto que elimina la necesidad de intercambiador de calor, reduciendo el costo. El aceite mineral tiene una presión de vapor más baja que el agua y es capaz de funcionar a altas temperaturas en forma líquida hasta $400{ }^{\circ} \mathrm{C}$; por otro lado, a diferencia de las sales fundidas, el aceite mineral no se congela durante la noche en las tuberías, lo cual excluye la necesidad de un sistema anticongelante [17]. Sin embargo, el aceite mineral es costoso en comparación con las sales fundidas y recientemente se han descubierto mezclas de sales fundidas con bajo punto de fusión, reemplazando el aceite mineral como material de almacenamiento de energía. La tendencia reciente en PCEST es utilizar sistemas indirectos donde el aceite mineral actúa como FTC y las mezclas salinas fundidas actúan como material de acumulación térmica.

\subsubsection{Sales fundidas}

Las sales fundidas son actualmente los materiales de almacenamiento de energía térmica más utilizados en las PCEST. Son baratas, especialmente los nitratos y su densidad es alta en comparación con otros medios fluidos de almacenamiento, lo que les da alta densidad de almacenamiento de energía. Las sales fundidas tienen una presión de vapor inferior a la del agua y son capaces de funcionar a altas temperaturas —en forma líquida hasta $400{ }^{\circ} \mathrm{C}-$, lo anterior permite el funcionamiento de la planta a altas temperaturas, por lo que mejora la eficiencia del ciclo termodinámico Rankine. Es necesario tener un punto de fusión inferior para las sales fundidas y cerca de la temperatura ambiente para que permanezcan líquidos durante el funcionamiento, también que el anticongelante sea mínimo durante la noche cuando no se dispone de energía solar. Pero las sales fundidas puras suelen tener puntos de fusión por encima de $200{ }^{\circ} \mathrm{C}$, lo cual es una desventaja; sin embargo, hoy en día la práctica es utilizar compuestos de sal (más mezclas de sal) que hacen bajar el punto de fusión por debajo de $100{ }^{\circ} \mathrm{C}$ y aun así tener una temperatura máxima superior a $500{ }^{\circ} \mathrm{C}$ [9], [18]. También pueden actuar como FTC, pero es más seguro tener un sistema anticongelante para hacer frente a cualquier riesgo de congelación; de lo contrario, se utilizará aceite mineral como FTC. Uno de los inconvenientes de las sales fundidas es que son agentes oxidantes y muy corrosivos, por lo que contenerlos a altas temperaturas es complicado. También su conductividad térmica es baja y tiene un cambio de volumen alrededor del $6 \%$ durante el proceso de fusión.

\subsubsection{Metales y aleaciones líquidas}

Los metales y aleaciones puras que tienen puntos de fusión bajos pero por encima de $300{ }^{\circ} \mathrm{C}$ también tienen potencial como medios de almacenamiento por calor sensibles, tienen una alta conductividad térmica y alta temperatura máxima de funcionamiento. Su presión de vapor es mínima, pero cuentan con la desventaja de un alto costo [19]. 


\subsubsection{Medio de almacenamiento sólido}

Los materiales sólidos de almacenamiento térmico tienen un bajo costo y alta disponibilidad. No tienen problema de presión de vapor. Toda vez que la presión de funcionamiento es la presión ambiente y, por lo tanto, no hay necesidad de contenedores presurizados, lo cual descarta los problemas de fugas. Debido a que no pueden circular fácilmente, solo puede hacerse almacenamiento pasivo de calor y se necesita un fluido, generalmente aire o agua como FTC para transportar calor dentro y fuera de materiales sólidos en un tanque de almacenamiento. Para aumentar la eficiencia de transferencia de calor hay un contacto directo entre el flujo de aire o agua y el medio de almacenamiento de calor en estado sólido, durante el proceso de carga y descarga [20]. Uno de los problemas con el almacenamiento sensible es que durante el proceso de descarga la temperatura del medio de almacenamiento disminuye, por lo que la temperatura del FTC también disminuye con el tiempo. Las aplicaciones a baja temperatura como el calentamiento de espacios y la recuperación de calor de residuos industriales pueden utilizar algunos de los materiales sólidos de almacenamiento de calor sensibles desarrollados a continuación.

\subsubsection{Rocas}

Pueden ser utilizados en un lecho compactado después de triturarlas a tamaños aproximados de $5 \mathrm{~cm}$ y emplear un FTC como el aire, agua o aceite mineral. El FTC caliente fluye entre las aberturas de las rocas compactadas y calienta la roca durante el proceso de carga, por otro lado, el FTC frío fluye entre las aberturas de las rocas y se calienta durante el proceso de descarga. Existe una gran superficie de contacto disponible para la transferencia de calor entre estos fluidos y las rocas, esto mejora la eficiencia de transferencia energética. Las rocas son conductores térmicos pobres y hay una pequeña área de contacto entre piezas de roca que minimiza la pérdida de calor durante el almacenamiento. Las rocas tienen ventajas como ser no tóxico, no inflamable, muy barato y fácilmente disponible. Los inconvenientes están en que estos sistemas demandan grandes caudales de FTC [21].

\subsubsection{Hormigón}

El concreto es un medio fácil de trabajar, pues posee una buena resistencia mecánica, no requiere de un recipiente de contención. Un enfoque de diseño para el intercambiador de calor entre el hormigón y el FTC es tener tuberías a través del bloque de hormigón, por medio de estas fluye el FTC. Uno de los problemas a considerar es que, a alta temperatura, se producen grietas después de ciclos repetidos de expansión y contracción térmica [22], [23].

\subsubsection{Arena}

Los materiales de grano pequeño disponibles comúnmente como grava o arena de sílice se pueden utilizar para el almacenamiento de energía térmica. Los granos de arena de sílice con un tamaño promedio de 0,2-0,5 mm pueden utilizarse en sistemas de almacenamiento de calor de lecho comprimido que utilizan aire como FTC, la densidad de embalaje es alta para los materiales de grano pequeño. La grava del basalto promedia los $0,4 \mathrm{~mm}$, esta puede ser utilizada directamente en el receptor solar para recoger la energía solar térmica cuando caen bajo la acción de la gravedad desde 
la parte superior de la torre del receptor solar. Después de absorber el calor de los rayos solares concentrados, durante su caída dentro de la torre, esta grava caliente se recoge en un pozo de almacenamiento aislado térmicamente. La temperatura de la grava caliente puede ir desde 700 a 1000 ${ }^{\circ} \mathrm{C}$, la cual es empleada para producir el vapor que acciona los turbos generadores eléctricos en el ciclo termodinámico Rankine. La contribución de [24] fue estudiar la posibilidad de utilizar arena en lecho comprimido como medio de almacenamiento de calor para el sistema de almacenamiento de energía térmica a alta temperatura, los resultados de esta investigación muestran que las arenas tienen potencial de almacenamiento para temperaturas de hasta $550{ }^{\circ} \mathrm{C}$.

\subsubsection{Ladrillos}

Los ladrillos en las paredes de edificios pueden almacenar energía térmica para minimizar los costos energéticos por concepto de calefacción de espacio [25], se pueden calentar en las horas punta durante la noche con electricidad más barata y almacenar el calor. El calor almacenado se extrae de los ladrillos durante el día por convección natural y radiación o por convección forzada utilizando un ventilador accionado eléctricamente [26], este calor almacenado mantiene el edificio caliente en las horas pico durante el día sin consumo de electricidad.

\subsection{Materiales de almacenamiento de energía térmica por calor latente}

Los materiales de almacenamiento por calor latentes también llamados materiales de cambio de fase (MCF) absorben la energía calorífica como su calor latente de fusión durante el proceso. En el trascurso del proceso de absorción de energía térmica se produce un cambio de fase y la oscilación de temperatura es muy pequeña. La energía térmica almacenada en el material puede expresarse como:

$$
Q=m * L
$$

Donde

$m$ : es la masa $(\mathrm{kg})$.

$L$ : es el calor latente de fusión $(\mathrm{kJ} / \mathrm{kg})$.

Por lo general, es el proceso de cambio de fase sólido a líquido el que se maneja. Las transformaciones de líquido a gas cuentan con un calor latente de cambio de fase más elevado; sin embargo, los monumentales cambios en el volumen de los materiales de almacenamiento asociados con la evaporación hacen que sea complejo el proceso de acumulación de energía y, por demás, poco práctico [27]. Si se utilizan materiales de almacenamiento de calor latente sólido a sólido, la falta de material líquido elimina el riesgo de fugas y no hay necesidad de encapsulación, pero su calor latente de transición es aproximadamente un orden de magnitud menor que el del material de almacenamiento de calor latente sólido a líquido, lo cual representa una considerable desventaja [28]. Los materiales de almacenamiento por calor latente deben contar con una alta conductividad térmica [29]-[31], deben tener un punto de temperatura de fusión próximo al rango de temperatura de funcionamiento requerido del sistema de AET, deben fundirse congruentemente con un subenfriamiento mínimo [32], ser químicamente estables, de bajo coste, no tóxicas y no corrosivas. 
Durante el proceso de descarga, la temperatura del medio de almacenamiento es constante, por lo que la temperatura del FTC también permanece estable con el tiempo, lo anterior es una ventaja sobre los materiales de almacenamiento por calor sensibles. Para materiales de almacenamiento de calor latente hay una menor diferencia de temperatura entre el almacenamiento y la liberación de calor [33]. El calor latente de fusión del material es muy grande en comparación con el calor específico, por ejemplo, la sal de nitrato de sodio tiene un calor especifico de $1,1 \mathrm{~kJ} / \mathrm{kg} * \mathrm{~K}$, pero su calor latente de fusión es de $172 \mathrm{~kJ} / \mathrm{kg}$ aproximadamente, esta gran diferencia les da a los materiales de almacenamiento por calor latente una ventaja, alta densidad de almacenamiento de energía. Se reduce entonces el volumen de los sistemas de AET, lo cual se traduce en que se reducen su superficie exterior y se minimizan la pérdida de calor.

\subsubsection{Materiales orgánicos}

Los materiales orgánicos de almacenamiento o acumulación térmica por calor latente y sus mezclas eutécticas han sido probados e implementados con éxito en muchas aplicaciones domésticas y comerciales, tales como refrigeración y aire acondicionado, calefacción de edificaciones, dispositivos electrónicos, calefacción solar de aire o agua, textiles, automóviles, alimentos y la industria aeroespacial. Los materiales orgánicos poseen la capacidad de fusión congruente sin separación de fases [19]. Los MCF orgánicos tienen características inherentes de baja conductividad térmica $\left(0,1-0,35 \mathrm{~W} / \mathrm{m}^{*} \mathrm{~K}\right)$, por lo que se requiere un área de superficie mayor para aumentar la tasa de transferencia de calor [18], [19]; además, debido al bajo punto de fusión de estos, todavía no se ha explorado su utilización para aplicaciones de alta temperatura como en centrales eléctricas.

\subsubsection{Parafinas}

Las parafinas consisten en una cadena de n-alcanos lineales o normales $\left(\mathrm{CH}_{3}-\left(\mathrm{CH}_{2}\right)-\mathrm{CH}_{3}\right)$ [34] Las ceras parafínicas comerciales son baratas con una densidad de almacenamiento térmico mode$\operatorname{rada}(200 \mathrm{~kJ} / \mathrm{kg})$ y un rango de temperaturas de fusión de $-10{ }^{\circ} \mathrm{C}$ hasta $67{ }^{\circ} \mathrm{C}$ [31], [35]. Son químicamente inertes y estables sin segregación de fase. Son no corrosivos, inodoros, duraderos, baratos, fácilmente disponibles, ecológicamente inofensivos y no tóxicos; sin embargo, tienen baja conductividad térmica $(0,2 \mathrm{~W} / \mathrm{m} * \mathrm{~K})$, esta característica limita sus aplicaciones [36]. La cera parafínica de calidad comercial se obtiene de la destilación del petróleo crudo. La mayoría de los MCF parafínicos son mezclas de hidrocarburos saturados con diferentes números de átomos de carbono en sus estructuras moleculares. La temperatura de fusión y el calor de fusión de los parafinos aumentan con la longitud media de la cadena de hidrocarburos, esta relación se puede emplear para diseñar las propiedades de los MCF mezclando parafinas físicamente diferentes. Incluso después de 1000 a 2000 ciclos, las ceras parafínicas de calidad comercial y otras parafinas puras tienen propiedades estables y una buena fiabilidad térmica; por otro lado, dado que no promueven la corrosión, son compatibles con recipientes metálicos, sin embargo, interactúan con algunos recipientes de plástico que tienen similitud química con estas. Polímeros como la poliolefina experimentan problemas como filtraciones y ablandamiento [37].

\subsubsection{2. Ácidos grasos}

Tienen una fórmula general de $\left(\mathrm{CH}_{2}\left(\mathrm{CH}_{3}\right)_{2 n}-\mathrm{COOH}\right)$ y tienen propiedades termofísicas ideales como material de almacenamiento de calor latente a baja temperatura. Los puntos de fusión y 
de ebullición de los ácidos grasos son relativamente altos comparados con las ceras parafínicas y los ácidos grasos saturados presentan cambios de volumen de transición de fase bajos. Tienen un comportamiento de fusión y congelación reproducible con muy poco o ningún superenfriamiento. Son más caros que los parafínicos, ligeramente corrosivos, tienen un olor desagradable y son combustibles [38]. En general, los puntos de fusión y congelación, el calor de fusión y el grado de cristalización de los ácidos grasos aumentan con el número de átomos de carbono en sus moléculas [35].

\subsubsection{Easters o ácidos grasos esenciales}

Se derivan de ácidos en los que un grupo hidroxilo (-OH) se sustituye por un grupo alquilo (-O). Los ácidos grasos Easters muestran una transición sólido-líquido en un rango de temperatura estrecho. Pueden formar los eutécticos sin o poco subenfriamiento. Las mezclas eutécticas de Easters tienen una temperatura de transición de fase cercana a la temperatura ambiente con alta entalpía de transición [39], [40]. Los ácidos grasos Easters tienen una buena disponibilidad, debido a sus aplicaciones comerciales en las industrias de polímeros, cosméticos y ropa inteligente.

\subsubsection{Alcoholes}

Los alcoholes de azúcar, también conocido como polialcoholes, se consideran como MCF de temperatura media entre 90 y $200{ }^{\circ} \mathrm{C}$. No han recibido mucha atención por parte de los investigadores. Estudios anteriores revelaron que los alcoholes como xilitol, eritritol y manitol poseen un calor latente de fusión cercano a $300 \mathrm{~kJ} / \mathrm{kg}$, el cual es mucho más alto que otros materiales de esta familia. Los alcoholes se han probado como posibles materiales de cambio de fase en las últimas décadas [41].

\subsubsection{Glicoles}

El polietilenglicol (PEG) tiene nombres diferentes como polioxietileno o óxido de polietileno. El PEG se compone de cadenas de éter dimetílico que tienen el grupo hidroxilo al final, con una fórmula de $\mathrm{HO}-\mathrm{CH}_{2}-\left(\mathrm{CH}_{2}-\mathrm{O}-\mathrm{CH}_{2}-\right) \mathrm{n}-\mathrm{CH}_{2}-\mathrm{OH}$. Son solubles tanto en agua como en compuestos orgánicos. El PEG viene en varios grados como PEG400, PEG600 etc., son química y térmicamente estables, no inflamables, no tóxicos, no corrosivos y económicos. El punto de fusión y el calor latente de fusión del PEG aumenta con el aumento del peso molecular [42]. Al igual que otros MCF orgánicos, el PEG también posee un de valor de conductividad térmica pequeño, presentado esta cualidad una desventaja.

\subsubsection{Materiales inorgánicos}

Estos materiales cuentan con una alta capacidad volumétrica de calor latente de alrededor de 350 $\mathrm{MJ} / \mathrm{m}^{3}$. Tienen propiedades estables incluso después de un gran número de ciclos térmicos y tienen una conductividad térmica relativamente alta de alrededor de $0,5 \mathrm{~W} / \mathrm{m} * \mathrm{~K}$; sin embargo, se funden incongruentemente [43], [44], son propensos a la segregación de fase y subenfriamiento. Otro problema importante con ellos es la corrosión que provocan las sales en los contenedores de metal. 


\subsubsection{Hidratos de sal}

Los hidratos de sal se utilizan normalmente a bajas temperaturas de funcionamiento en el intervalo de 30 a $50{ }^{\circ} \mathrm{C}$. Tienen una fórmula general $\mathrm{AB} \cdot \mathrm{nH}_{2} \mathrm{O}$, son sales inorgánicas que contienen agua de cristalización. Durante la transformación de estado, se produce la deshidratación de la sal [45]. Esto puede ocurrir de dos maneras. Podría haber un hidrato de sal que contiene menos moléculas de agua o podría haber formas anhidras de la sal. Dependiendo del comportamiento de fusión, los hidratos de sal pueden clasificarse como se muestra a continuación.

- Hidratos de sales congruentes: la sal es soluble en el agua de hidratación durante la fusión.

- Hidratos de sal incongruentes: la sal es parcialmente soluble en el agua de hidratación durante la fusión.

- Hidratos de sal semicongruentes: después del cambio de fase, las fases sólida y líquida tienen diferentes composiciones debido a la transformación del hidrato de sal, en un hidrato de salino con una menor cantidad de agua.

Sin embargo, un gran número de hidratos de sal que tienen el potencial de convertirse en MCF con temperatura de fusión adecuada y un gran calor latente se funden de forma incongruente. El agua liberada durante la transición de fase no disuelve la sal cristalina formada durante el proceso de deshidratación, lo anterior debido a la diferencia de densidad entre la sal, la separación de fases de agua y la sedimentación que se produce en los contenedores; este es un serio problema técnico en aplicaciones prácticas. Uno de los métodos utilizados para prevenir la segregación y la sedimentación es la adición de agentes gelificantes o espesantes [35]. La adición de material gelificante polimérico o celulósico a la sal impide la sedimentación de la sal, como agente espesante aumenta la viscosidad del hidrato de sal y ayuda a mantener las moléculas de hidrato de sal juntas. Otra desventaja de los hidratos de sal es un significativo superenfriamiento. Esto se debe a su pobre capacidad de nucleación y, por tanto, para evitar este problema, se añaden agentes nucleantes. Los agentes nucleantes como el bórax, el carbono [31], [46], etc., ayudan a superar el superenfriamiento, pero reducen la velocidad de transferencia de calor al disminuir la conductividad térmica.

\subsubsection{Sales}

Las sales inorgánicas operan similar a las sales fundidas explicadas con anterioridad, donde se usan sales de temperaturas de fusión relativamente más bajas como medio de almacenamiento de líquido sensible, pero también pueden actuar como materiales latentes de almacenamiento de calor para aplicaciones a altas temperaturas por encima de los $100^{\circ} \mathrm{C}$. Caen en muchos subgrupos de materiales como nitratos, carbonatos, hidróxidos, cloruros, etc., y tienen una amplia gama de temperaturas de fusión [47].

\subsubsection{Metales y aleaciones metálicas}

Los metales y sus aleaciones cuentan con altos valores de conductividad térmica, buena estabilidad térmica y fiabilidad [2], [48]. Poseen un calor de transición de fase elevado por unidad de volumen o unidad de masa, por lo tanto, tienen una capacidad de almacenamiento de energía muy 
alta. Tienen un cambio considerable en el volumen durante el cambio de fase y una presión de vapor despreciable.; sin embargo, cuando el volumen es una prioridad, son capaces de competir con las sales [27], [36].

\subsubsection{Eutéctico}

El eutéctico es una composición de dos o más componentes tales como orgánico-orgánico, orgánicoinorgánico e inorgánico-inorgánico, cada uno de ellos cambia su fase de forma congruente, es decir, tiene un punto de fusión o congelación definido y forma una mezcla de cristal componente durante la cristalización. Los eutécticos generalmente se funden y congelan congruentemente y no dejan posibilidades de separación de los componentes [1].

\subsection{Materiales de cambio de fase compuestos}

Para solucionar el problema de la mala conductividad térmica, frecuentemente se dispersan partículas de alta conductividad como lo es el carbono, el grafito o los MCF, tales mezclas se denominan materiales compuestos de cambio de fase, estos se pueden preparar usando tanto materiales de cambio de fase orgánicos como inorgánicos. Los materiales térmicos conductores adicionales deben ser compatibles con el MCF.

\subsubsection{Compuestos de grafito}

El grafito puede usarse en diferentes formas como se muestra a continuación. Los trabajos de [49], [50] expusieron el rendimiento de los $\mathrm{MCF}$ eutécticos $\mathrm{NaNO}_{3} / \mathrm{KNO}_{3}$ con grafito. El grafito incrementa la conductividad térmica, pues es dispersado dentro de la sal fundida. Esta práctica mejoró la conductividad térmica catorce veces hasta una conductividad efectiva de $9 \mathrm{~W} / \mathrm{m} * \mathrm{~K}$. Las contribuciones de [51], [52] mostraron el desempeño de los MCF compuestos por n-docosanol parafínicos con grafito expandido. Las conductividades térmicas de los MCF compuesto con fracción de masa de grafito expandido al $2 \%, 4 \%, 7 \%$ y $10 \%$ indicaron que la conductividad térmica de la parafina que es de $\left(0,22 \mathrm{~W} / \mathrm{m}^{*} \mathrm{~K}\right)$ aumentó en $81,2 \%, 136,3 \%, 209,1 \%$ y 272,7\%, respectivamente.

\subsubsection{Grafito natural}

Tienen una estructura cristalina bien alineada y una alta conductividad térmica. Han apilado láminas de carbono, donde los átomos de carbono se mantienen unidos por fuertes enlaces covalentes y estas hojas apiladas se mantienen unidas por enlaces débiles de Van der Waals, por lo tanto, cuentan con una elevada densidad. Requieren un tratamiento mínimo durante la producción y su costo es muy bajo [53].

\subsubsection{Grafito natural expandido}

Esto se produce a partir de grafito natural después de someterse a dos procesos, tratamiento químico y exfoliación térmica. En reacciones con diversos ácidos, se superan los enlaces débiles y se incorporan moléculas del reactivo entre las capas del grafito. La estructura de grafito tiene que expandirse para acomodar las moléculas reaccionantes, pero la estructura laminar permanece intacta, 
esto se llama un compuesto intercalado. Después de que el compuesto intercalado con grafito se lava en agua y se seca, se calienta para provocar una rápida expansión de las capas de grafito. El tratamiento térmico se realiza en una atmósfera de aire a temperaturas superiores a $500{ }^{\circ} \mathrm{C}$. El agua se incorpora entre las capas de carbono durante la formación del compuesto intercalado. La evaporación casi instantánea del agua durante el proceso de tratamiento térmico es responsable de la expansión. Cada partícula de grafito natural se modifica en forma de gusano en el que las capas de grafeno se diseminan conduciendo a una gran porosidad, estos gusanos pueden hacerse en un lecho y luego comprimirse para formar una matriz de grafito consolidada de alta porosidad y conductividad térmica [54].

\subsubsection{Polvo de grafito expandido}

Esto se prepara moliendo el grafito expandido en polvo. Tiene una estructura menos compacta que el grafito natural y tiende a construir redes incluso en pequeñas concentraciones. Así, en comparación con el grafito natural, se requieren cantidades menores de polvo de grafito expandido para alcanzar una conductividad térmica similar. Sin embargo, debido a los correspondientes tratamientos necesarios para su producción, es más caro que el grafito natural [36].

\subsubsection{Nano compuesto}

Se emplean diferentes nano-estructuras como aditivos de mejora térmica en MCF. La contribución de [55], [56] fue que investigaron varios tipos de nanopartículas y la implantación de estas en sistemas de acumulación térmica. Las nanopartículas tienen la ventaja de ser extremadamente pequeñas en tamaño, por lo tanto, se comportan como fluido en depósitos y conductos. Generalmente, las mejoras térmicas conseguidas con nanoestructuras están enfocadas en la conductividad térmica. Los trabajos de [57]-[59] contienen una revisión de los actuales estudios experimentales sobre las variaciones en las propiedades termofísicas de MCF debido a la dispersión de nanopartículas. Los tipos de nano-partículas son los siguientes:

- Nanofibras basadas en carbono, nano plaquetas, grafeno y nanotubos de carbono.

- Metálico (Ag, Al, C / Cu y Cu).

- Óxido metálico $\left(\mathrm{Al}_{2} \mathrm{O}_{3}, \mathrm{CuO}, \mathrm{NiO}, \mathrm{SiO}_{2}, \mathrm{ZnO}, \mathrm{MgO}\right.$ y $\left.\mathrm{TiO}_{2}\right)$.

- Nano-alambres de plata.

\subsubsection{Compuesto de forma estable}

Un compuesto de forma estable se considera un MCF, si puede mantener la misma forma en estado sólido incluso cuando la temperatura del material compuesto es superior a la temperatura de fusión del MCF. En un compuesto de forma estable, el MCF se puede moldear a formas requeridas con una proporción de área superficial a volumen más alta sin la ayuda de una encapsulación para aumentar la velocidad de transferencia de calor. Aquí la idea principal es mejorar la superficie de contacto del MCF, el aumento de la conductividad térmica no es la prioridad. En los estudios de [60], [61] se analizaron compuestos de polietileno parafínico de alta densidad, estables en forma, donde el parafínico actúa como material de acumulación térmica por calor latente, mientras que el 
polietileno de alta densidad actúa como material de soporte que da resistencia estructural para evitar la fuga de partículas fundidas. Esta práctica generó mejoras del $24 \%$ en la conductividad térmica.

\subsubsection{Materiales de Cambio de Fase Microencapsulados (MCFME)}

Las microcápsulas se pueden describir como partículas que contienen material de núcleo rodeado por un revestimiento o envoltura y tienen diámetros en el intervalo de 1-1000 $\mu \mathrm{m}$. La microencapsulación es ampliamente utilizada en aplicaciones comerciales, incluyendo textiles, adhesivos, cosméticos, fármacos y edificaciones [34]. Los materiales de cambio de fase microencapsulados (MCFME) deben tener morfología requerida, diámetro uniforme, resistencia mecánica de la envoltura, capacidad de penetración y estabilidad térmica. Las bolsas, tubos, esfera, paneles u otros recipientes que contienen MCFME actúan directamente como intercambiadores de calor. El MCFME también se puede incorporar a materiales de construcción. La carcasa contiene el MCF líquido y evita cambios en su composición debido al contacto con el medio ambiente. Aumenta la superficie de contacto para la transferencia de calor, también añade la estabilidad mecánica a MCFME, si la envoltura es suficientemente rígida. Los MCFME están disponibles en varias formas y tamaños. La microencapsulación es un proceso relativamente costoso. La resina de urea-formaldehído, resina de melamina-formaldehído y poliuretanos son los materiales de concha de encapsulación más frecuentes en los sistemas de acumulación de energía térmica [62].

\subsubsection{Coacervación}

Cuando dos o más coloides de carga opuesta se dispersan en una solución acuosa de un MCF, la coacervación se produce debido a la fuerza electrostática entre partículas coloidales cargadas de forma opuesta para formar dos nuevas fases, una rica y una pobre en concentración coloidal. Estas partículas coloidales concentradas forman gotitas esféricas unidas entre sí por fuerzas electrostáticas [63], [64].

\subsubsection{Polimerización en suspensión}

Las microcápsulas con una cubierta de polímero y un núcleo de MCF pueden obtenerse mediante un proceso basado en la polimerización en suspensión. En este procedimiento, se dispersa un monómero o una mezcla de monómeros que contienen un iniciador en una solución acuosa de MCF empleado agitación mecánica. El iniciador puede descomponerse a radicales libres por calentamiento, y el radical libre desencadena entonces la polimerización de monómeros. El tamaño de las gotitas de las microcápsulas está controlado por parámetros físicoquímicos (viscosidad, densidades y tensión interfacial), parámetros del equipo (porosidad, diámetro de poro, diámetro del tubo y dimensión del agitador) y las condiciones de funcionamiento (caudal, presión) [64].

\subsubsection{Polimerización en emulsión}

Esto implica una emulsión en la que un monómero se emulsiona con tensioactivos en una fase acuosa. Las gotas líquidas de MCF que actúan como fase oleosa se dispersan en la solución acuosa. Los tensioactivos que tienen lados hidrófobos e hidrófilos se alinean alrededor de la interface entre monómeros en solución acuosa y gotitas líquidas del MCF. La parte hidrófila entra en contacto 
con la solución acuosa y la parte hidrófoba entra en contacto con la gotita de líquido del MCF, lo anterior da como resultado el iniciador que desencadena la polimerización de las moléculas de monómero alrededor de la gotita esférica. Tales gotitas de emulsión con conchas de polímero formadas a lo largo de la solución acuosa dan como resultado una emulsión con hasta un $50 \%$ de concentración de MCF microencapsulada suspendida [65], [66].

\subsubsection{Poliadición}

Este tipo de método de formación de microcápsulas rellenas con MCF se basa en la reacción de poliadición interfacial entre un monómero $(\sigma)$ en la fase oleosa y un monómero $(\tau)$ en correspondencia en la fase acuosa, esto puede implicar la dispersión de una solución en fase oleosa del MCF y el monómero $(\sigma)$ en otra solución en fase acuosa que contiene el mononer $(\tau)$, añadiendo luego un iniciador para generar la reacción de poliadición entre el monómero $(\sigma)$ y el mononer $(\tau)$. Una membrana de poliurea se forma casi instantáneamente en la superficie de las gotitas de la fase de aceite dispersada de la MCF [2].

\subsection{Materiales termoquímicos de almacenamiento de calor}

El sistema termoquímico de almacenamiento térmico [17], [67], [68] utiliza una reacción química reversible. La energía térmica almacenada es igual a la entalpía de reacción. Durante el proceso de carga, una reacción endotérmica hacia adelante absorbe el calor y la energía térmica absorbida se utiliza para disociar un reactivo químico $\left(\mathrm{X}_{0}\right)$ en los productos $\left(\mathrm{X}_{1}\right)$ y $\left(\mathrm{X}_{2}\right)$. Durante el proceso de descarga, los reactantes $\left(\mathrm{X}_{1}\right)$ y $\left(\mathrm{X}_{2}\right)$ experimentan una reacción exotérmica hacia atrás que produce $\left(\mathrm{X}_{0}\right)$, liberando calor en este proceso [69]. Los productos de ambas reacciones se pueden almacenar ya sea a temperatura ambiente o a temperatura de trabajo. La energía térmica almacenada en el material termoquímico puede expresarse como:

$$
Q=n * \Delta H
$$

Donde

$n$ : es el número molar del reactivo (mol).

$\Delta H$ : es la entalpía de reacción $(\mathrm{kJ} / \mathrm{mol})$.

La tecnología de almacenamiento termoquímico se encuentra todavía en fase de laboratorio, mientras que las tecnologías de calor sensible y latente están maduras y se encuentran en fase industrial. La densidad de energía almacenada en este proceso termoquímico es mayor comparada con los materiales de almacenamiento de calor sensibles y latentes. Los sistemas de acumulación térmica por calor sensibles, calor latente y termoquímico tienen una densidad volumétrica aproximada de almacenamiento de energía de 50,100 y $500 \mathrm{~kW} * \mathrm{~h} / \mathrm{m}^{3}$, respectivamente. La duración de la carga térmica es limitada en el caso de materiales de almacenamiento de calor sensibles y latentes debido a pérdidas térmicas. En el caso de almacenamiento termoquímico, es teóricamente ilimitada debido a pérdidas térmicas mínimas. La complejidad de la tecnología de acumulación térmica mediante un proceso termoquímico es considerable, frente a los procesos de almacenamiento por calor sensibles y latentes [70]. El primer paso para desarrollar un sistema AET termoquímico es la selección de la reacción y el estudio de sus características químicas y físicas, tales como la reversibilidad, la velocidad de reacción, las condiciones de funcionamiento (presión y temperatura) 
y las propiedades cinéticas [1]. Los requisitos de un material de almacenamiento termoquímico adecuado son los siguientes:

- La reacción endotérmica utilizada para el almacenamiento de calor debe ocurrir a una temperatura por debajo de $1273 \mathrm{~K}$.

- La reacción exotérmica utilizada para recuperar el calor debe ocurrir a una temperatura por encima de $773 \mathrm{~K}$.

- Las reacciones en ambos sentidos deben ser completamente reversibles sin reacciones secundarias.

- La gran entalpía de reacción y el pequeño volumen molar pueden maximizar la capacidad de almacenamiento de energía térmica.

- Los compuestos químicos de ambas reacciones se pueden manejar fácilmente.

- Los compuestos químicos no deben reaccionar con el medio ambiente.

\section{Discusión}

El empleo de la energía solar térmica representa una fuente de energía renovable cada vez más atractiva; sin embargo, uno de los factores clave que determinan el desarrollo de esta tecnología es su integración a sistemas de almacenamiento de energía térmica, eficientes y rentable para superar el carácter intermitente de la luz solar y ser más económicamente competitivos [71], [72]. El considerable incremento en el número de investigaciones y patentes vinculadas con esta tecnología en los últimos años da fe del protagonismo de la acumulación térmica como práctica energética.

Un sistema AET consta de tres partes: medio de almacenamiento, intercambiador de calor y tanque de almacenamiento. El medio de almacenamiento puede operar por calor sensible, calor latente o mediante un proceso termoquímico [48], [73]. El propósito del intercambiador de calor es suministrar o extraer calor del medio de almacenamiento. El tanque de almacenamiento contiene el medio de almacenamiento y aísla el proceso del entorno. Los sistemas AET deben ser diseñados para cumplir con ciertos criterios, que dependen del tipo, tamaño y diseño de la aplicación [1], [37], [74].

Antes de elegir un sistema AET adecuado, es necesario realizar un análisis exhaustivo de todos los requisitos. El sistema AET debe ser compatible con todas las otras unidades de la instalación, además, debe ceñirse a la estrategia operativa global de la instalación como el rango de temperatura de funcionamiento, el número de horas de acumulación térmica demandado, la tasa de carga y descarga, la integración con el sistema de recolección solar, etc. Debe garantizarse su estabilidad en un gran número de ciclos, que tanto el contenedor como el medio de almacenamiento puedan soportar sin degradación de sus propiedades. La estabilidad a largo plazo del sistema puede verse comprometida por varios factores, una escasa estabilidad de las propiedades de los materiales sometidos a ciclos térmicos extensivos o corrosión e incompatibilidad química entre el MCF y su recipiente; por consiguiente, se debe elegir el material de almacenamiento más adecuado, el intercambiador 
de calor entre el material de almacenamiento térmico y el fluido de transferencia de calor [9]. En el almacenamiento de energía térmica, actualmente las principales áreas de enfoque son la reducción de costos del material de almacenamiento, la reducción de costos de operación y la mejora en la vialidad económica del almacenamiento de energía.

\section{Conclusiones}

Las aplicaciones para el AET pueden clasificarse como áreas de alta, media y baja temperatura. En el lado de alta temperatura, los materiales inorgánicos como las sales de nitrato son los materiales de almacenamiento de energía térmica más utilizados, mientras que en el lado medio y bajo, los materiales orgánicos como la parafina comercial son los más utilizados. La mejora de la conductividad térmica de los materiales de almacenamiento de energía térmica es un área de enfoque importante en las actuales investigaciones. Se están explorando tecnologías de fabricación rentables para MCF microencapsulado y materiales compuestos. La optimización de las propiedades termofísicas como el punto de fusión de los materiales de almacenamiento de energía térmica, se explora con técnicas como las mezclas eutécticas y la longitud de la cadena de hidrocarburos, etc. Para materiales de almacenamiento por calor sensibles y latentes, las tecnologías son bastante maduras y difundidas. Los materiales termoquímicos están todavía en fase de laboratorio, estos tienen un gran potencial como materiales de almacenamiento de energía térmica en el futuro debido a su gran capacidad de almacenamiento de energía por unidad de volumen.

\section{Referencias}

[1] P. A. J. Donkers, L. C. Sögütoglu, H. P. Huinink, H. R. Fischer, and O. C. G. Adan, "A Review of Salt Hydrates for Seasonal Heat Storage in Domestic Applications", Applied Energy, Vol. 199, pp. 45-68, 2017. $\uparrow 145,156,160$

[2] B. Kanimozhi, B. R. Ramesh Bapu, and V. Pranesh, "Thermal Energy Storage System Operating with Phase Change Materials for Solar Water Heating Applications: Doe Modelling", Applied Thermal Engineering, Vol. 123, pp. 614-624, 2017. $\uparrow 145,155,159$

[3] G. Li and X. Zheng, "Thermal Energy Storage System Integration Forms for a Sustainable Future", Renewable and Sustainable Energy Reviews, Vol. 62, pp. 736- 757, 2016. $\uparrow 145,146$

[4] J. Arévalo, F. Santos, and S. Rivera, "Application of Analytical Uncertainty Costs of Solar, Wind and Electric Vehicles in Optimal Power Dispatch“, Ingeniería, Vol. 22, No. 3, pp. 152-161, 2017. $\uparrow 145$

[5] J. D. B. Rodríguez, C. A. Ramos-Paja, and E. F. Mejía, "Modeling and Parameter Calculation of Photovoltaic Fields in Irregular Weather Conditions", Ingeniería, Vol. 17, No. 1, pp. 37-48, 2012. $\uparrow 145$

[6] S. Pintaldi, S. Sethuvenkatraman, S. White, and G. Rosengarten, "Energetic Evaluation of Thermal Energy Storage Options for High Efficiency Solar Cooling Systems“", Applied Energy, Vol. 188, pp. 160-177, $2017 \uparrow 145$

[7] H. Shamsi, M. Boroushaki, and H. Geraei, "Performance Evaluation and Optimization of Encapsulated Cascade Pcm Thermal Storage“, Journal of Energy Storage, Vol. 11, pp. 64-75, 2017. $\uparrow 145$

[8] J. A. G. Moreno, C. L. T. Rodríguez, and R. A. P. Suesca, "Generación Híbrida de Energía Eléctrica como Alternativa para Zonas no Interconectadas“, Ingeniería, Vol. 12, No. 1, pp. 57-63, 2006. 1145

[9] F. Kleiner, K. Posern, and A. Osburg, "Thermal Conductivity of Selected Salt Hydrates for Thermochemical Solar Heat Storage Applications Measured by the Light Flash Method",Applied Thermal Engineering, Vol. 113, pp. 1189-1193, 2017. $\uparrow 146,150,161$

[10] J. F. Burnham, "Scopus Database: a Review“, Biomedical Digital Libraries, Vol. 3, No. 1, P. 1, 2006. $\uparrow 147$

[11] J. I. Beltrán, J. Wang, F. Montero-Chacón, and Y. Cui, "Thermodynamic Modeling of Nitrate Materials for Hybrid Thermal Energy Storage: Using Latent and Sensible Mechanisms“, Solar Energy, Vol. 155, pp. 154-166, 2017. $\uparrow$ 149 
[12] A. Gil Et Al., "State of The Art on High Temperature Thermal Energy Storage for Power Generation. Part 1-Concepts, Materials and Modellization“, Renewable and Sustainable Energy Reviews, Vol. 14, No. 1, pp. 31-55, 2010. $\uparrow 149$

[13] S. M. Hasnain, "Review On Sustainable Thermal Energy Storage Technologies, Part I: Heat Storage Materials and Techniques“, Energy Conversion and Management, Vol. 39, No. 11, pp. 1127-1138, 1998. 149

[14] G. Murali and K. Mayilsamy, "Effect of Latent Thermal Energy Storage and Inlet Locations on Enhancement of Stratification in a Solar Water Heater Under Discharging Mode“, Applied Thermal Engineering, Vol. 106, pp. 354-360, 2016. $\uparrow 149$

[15] T. Kousksou, P. Bruel, A. Jamil, T. El Rhafiki, and Y. Zeraouli, "Energy Storage: Applications and Challenges“, Solar Energy Materials and Solar Cells, Vol. 120, pp. 59- 80, 2014. $\uparrow 150$

[16] S. Zou and X. Xie, "Simplified Model for Coefficient of Performance Calculation of Surface Water Source Heat Pump“, Applied Thermal Engineering, Vol. 112, pp. 201- 207, 2017. $\uparrow 150$

[17] J. Lizana, R. Chacartegui, A. Barrios-Padura, and J. M. Valverde, "Advances in Thermal Energy Storage Materials and Their Applications Towards Zero Energy Buildings: a Critical Review“, Applied Energy, Vol. 203, pp. 219-239, 2017. $\uparrow 150,159$

[18] M. Liu, W. Saman, and F. Bruno, "Review on Storage Materials and Thermal Performance Enhancement Techniques for High Temperature Phase Change Thermal Storage Systems", Renewable and Sustainable Energy Reviews, Vol. 16, No. 4, pp. 2118-2132, 2012. $\uparrow 150,153$

[19] B. Xu, P. Li, and C. Chan, "Application of Phase Change Materials for Thermal Energy Storage in Concentrated Solar Thermal Power Plants: a Review to Recent Developments“, Applied Energy, Vol. 160, pp. 286-307, 2015. $\uparrow$ 150,153

[20] L. Nkhonjera, T. Bello-Ochende, G. John, and C. K. King'ondu, "A Review of Thermal Energy Storage Designs, Heat Storage Materials and Cooking Performance of Solar Cookers with Heat Storage", Renewable and Sustainable Energy Reviews, Article in Press 2016. $\uparrow 151$

[21] M. Hänchen, S. Brückner, and A. Steinfeld, "High-Temperature Thermal Storage Using a Packed Bed of Rocks-Heat Transfer Analysis and Experimental Validation“, Applied Thermal Engineering, Vol. 31, No. 10, pp. 1798-1806, 2011. $\uparrow 151$

[22] M. Martins, U. Villalobos, T. Delclos, P. Armstrong, P. G. Bergan, and N. Calvet, "New Concentrating Solar Power Facility for Testing High Temperature Concrete Thermal Energy Storage“, In Energy Procedia, Vol. 75, pp. 2144-2149, 2015. $\uparrow 151$

[23] V. A. Salomoni Et Al., "Thermal Storage of Sensible Heat Using Concrete Modules in Solar Power Plants", Solar Energy, Vol. 103, pp. 303-315, 2014. $\uparrow 151$

[24] D. Schlipf, P. Schicktanz, H. Maier, and G. Schneider, "Using Sand and Other Small Grained Materials as Heat Storage Medium in a Packed Bed Httess", Energy Procedia, Vol. 69, pp. 1029-1038, 2015. $\uparrow 152$

[25] D. Zhitomirsky, E. Cho, and J. C. Grossman, "Solid-State Solar Thermal Fuels for Heat Release Applications", Advanced Energy Materials, Vol. 6, No. 6, Art. No. 1502006, pp. 98-113, 2016. $\uparrow 152$

[26] S. Krishnan and B. Sivaraman, "Experimental Investigations On Thermal Storage in a Solar Dryer", International Energy Journal, Vol. 17, No. 1, pp. 23-35, 2017. $\uparrow 152$

[27] B. Cárdenas and N. León, "High Temperature Latent Heat Thermal Energy Storage: Phase Change Materials, Design Considerations and Performance Enhancement Techniques", Renewable and Sustainable Energy Reviews, Vol. 27, pp. 724-737, 2013. $\uparrow 152,156$

[28] G. Cáceres, M. Montané, S. Nasirov, and R. O'ryan, "Review of Thermal Materials for Csp Plants and Lcoe Evaluation for Performance Improvement Using Chilean Strategic Minerals: Lithium Salts and Copper Foams“, Sustainability (Switzerland), Vol. 8, No. 2, Art. No. 106, pp. 62-77, 2016. $\uparrow 152$

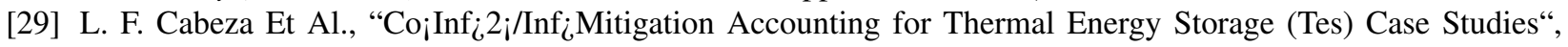
Applied Energy, Vol. 155, pp. 365-377, 2015. $\uparrow 152$

[30] P. Marin Et Al., "Energy Savings Due To The Use of Pcm for Relocatable Lightweight Buildings Passive Heating and Cooling in Different Weather Conditions", Energy and Buildings, Vol. 129, pp. 274-283, 2016. $\uparrow 152$

[31] M. M. Farid, A. M. Khudhair, S. A. K. Razack, and S. Al-Hallaj, "A Review On Phase Change Energy Storage: Materials and Applications“, Energy Conversion and Management, Vol. 45, No. 9, pp. 1597-1615, 2004. $\uparrow 152$, 153,155

[32] W. Su, J. Darkwa, and G. Kokogiannakis, "Review of Solid-Liquid Phase Change Materials and Their Encapsulation Technologies“, Renewable and Sustainable Energy Reviews, Vol. 48, pp. 373-391, 2015. $\uparrow 152$

[33] H. Akeiber Et Al., "A Review On Phase Change Material (Pcm) for Sustainable Passive Cooling in Building 
Envelopes“, Renewable and Sustainable Energy Reviews, Vol. 60, pp. 1470-1497, 2016. $\uparrow 153$

[34] R. Sharma, P. Ganesan, V. Tyagi, H. Metselaar, and S. Sandaran, "Developments in Organic Solid-Liquid Phase Change Materials and Their Applications in Thermal Energy Storage", Energy Conversion and Management, Vol. 95, pp. 193-228, 2015. $\uparrow 153,158$

[35] K. Pielichowska and K. Pielichowski, "Phase Change Materials for Thermal Energy Storage“, Progress in Materials Science, Vol. 65, pp. 67-123, 2014. $\uparrow 153,154,155$

[36] H. Ghasemi Bahraseman, E. M. Languri, and J. East, "Fast Charging of Thermal Energy Storage Systems Enabled by Phase Change Materials Mixed with Expanded Graphite“, International Journal of Heat and Mass Transfer, Vol. 109, pp. 1052-1058, 2017. $1153,156,157$

[37] P. Feliński and R. Sekret, "Experimental Study of Evacuated Tube Collector/Storage System Containing Paraffin As a Pcm“, Energy, Vol. 114, pp. 1063-1072, 2016. $\uparrow 153,160$

[38] R. K. Sharma, P. Ganesan, V. V. Tyagi, H. S. C. Metselaar, and S. C. Sandaran, "Developments in Organic Solid-Liquid Phase Change Materials and Their Applications in Thermal Energy Storage", Energy Conversion and Management, Vol. 95, pp. 193- 228, 2015. $\uparrow 154$

[39] F. J. Ruiz-Cabañas, A. Jové, C. Prieto, V. Madina, A. I. Fernández, and L. F. Cabeza, "Materials Selection of Steam-Phase Change Material (Pcm) Heat Exchanger for Thermal Energy Storage Systems in Direct Steam Generation Facilities“, Solar Energy Materials and Solar Cells, Vol. 159, pp. 526-535, 2017. $\uparrow 154$

[40] A. Safari, R. Saidur, F. Sulaiman, Y. Xu, and J. Dong, "A Review On Supercooling of Phase Change Materials in Thermal Energy Storage Systems", Renewable and Sustainable Energy Reviews, Vol. 70, pp. 905-919, 2017. $\uparrow 154$

[41] E. P. Del Barrio, R. Cadoret, J. Daranlot, and F. Achchaq, "New Sugar Alcohols Mixtures for Long-Term Thermal Energy Storage Applications at Temperatures Between $70^{\circ} \mathrm{C}$ and $100^{\circ} \mathrm{C}$ ", Solar Energy Materials and Solar Cells, Vol. 155, pp. 454-468, 2016. $\uparrow 154$

[42] N. Sarier and E. Onder, "Organic Phase Change Materials and Their Textile Applications: an Overview", Thermochimica Acta, Vol. 540, pp. 7-60, 2012. $\uparrow 154$

[43] A. Gutierrez Et A1., "Characterization of Wastes Based On Inorganic Double Salt Hydrates as Potential Thermal Energy Storage Materials", Solar Energy Materials and Solar Cells, Vol. 170, pp. 149-159, 2017. $\uparrow 154$

[44] S. A. Mohamed Et Al., "A Review on Current Status and Challenges of Inorganic Phase Change Materials for Thermal Energy Storage Systems“, Renewable and Sustainable Energy Reviews, Vol. 70, pp. 1072-1089, 2017. $\uparrow$ 154

[45] J. Trahan, S. Kuravi, D. Y. Goswami, M. Rahman, and E. Stefanakos, "Thermal Characterization of High Temperature Inorganic Phase Change Materials for Thermal Energy Storage Applications", In Asme 2012 6th International Conference on Energy Sustainability, ES 2012, Collocated with The Asme 2012 10th International Conference On Fuel Cell Science, Engineering and Technology, pp. 623-630. San Diego: American Society of Mechanical Engineers, pp. 41-56, 2012. $\uparrow 155$

[46] F. Guarino, A. Athienitis, M. Cellura, and D. Bastien, "PCM Thermal Storage Design in Buildings: Experimental Studies and Applications to Solaria in Cold Climates", Applied Energy, Vol. 185, pp. 95-106, 2017. $\uparrow 155$

[47] D. Zhang and X. Ke, "Study of Phase Change Energy Storage Technology Development and Policy of PeakValley Electric Price“, Energy Education Science and Technology Part A: Energy Science and Research, Vol. 32, No. 5, pp. 3993-4004, 2014. $\uparrow 155$

[48] G. Alva, L. Liu, X. Huang, and G. Fang, "Thermal Energy Storage Materials and Systems for Solar Energy Applications“, Renewable and Sustainable Energy Reviews, Vol. 68, pp. 693-706, 2017. $\uparrow 155,160$

[49] S. Pincemin, R. Olives, X. Py, and M. Christ, "Highly Conductive Composites Made of Phase Change Materials and Graphite for Thermal Storage“, Solar Energy Materials and Solar Cells, Vol. 92, No. 6, pp. 603-613, 2008. $\uparrow$ 156

[50] C. Zhu and B. Li, "Research Status of Phase Change Thermal Storage Material Applied for Solar Heating", Materials China, Vol. 36, No. 3, pp. 236-240, 2017. $\uparrow 156$

[51] A. Karaipekli and A. Sari, "Development and Thermal Performance of Pumice/Organic Pcm/Gypsum Composite Plasters for Thermal Energy Storage In Buildings“, Solar Energy Materials and Solar Cells, Vol. 149, pp. 19-28, 2016. $\uparrow 156$

[52] A. Sarı and A. Karaipekli, "Thermal Conductivity and Latent Heat Thermal Energy Storage Characteristics of Paraffin/Expanded Graphite Composite as Phase Change Material“, Applied Thermal Engineering, Vol. 27, No. 8, pp. 1271-1277, 2007. $\uparrow 156$

[53] T. Xu, Y. Li, J. Chen, and J. Liu, "Preparation and Thermal Energy Storage Properties of Lino3-KclNano3/Expanded Graphite Composite Phase Change Material“, Solar Energy Materials and Solar Cells, Vol. 169, 
pp. 215-221, 2017. 1156

[54] X. Huang, G. Alva, L. Liu, and G. Fang, "Preparation, Characterization and Thermal Properties of Fatty Acid Eutectics/Bentonite/Expanded Graphite Composites as Novel Form-Stable Thermal Energy Storage Materials“, Solar Energy Materials and Solar Cells, Vol. 166, pp. 157-166, $2017 \uparrow 157$

[55] C. D. S. Brites Et Al., "Tethering Luminescent Thermometry and Plasmonics: Light Manipulation To Assess Real-Time Thermal Flow in Nanoarchitectures“, Nano Letters, Vol. 17, No. 8, pp. 4746-4752, 2017. $\uparrow 157$

[56] J. Khodadadi, L. Fan, and H. Babaei, "Thermal Conductivity Enhancement of Nanostructure-Based Colloidal Suspensions Utilized as Phase Change Materials for Thermal Energy Storage: a Review“, Renewable and Sustainable Energy Reviews, Vol. 24, pp. 418-444, 2013. $\uparrow 157$

[57] M. Kibria, M. Anisur, M. Mahfuz, R. Saidur, and I. Metselaar, "A Review on Thermophysical Properties of Nanoparticle Dispersed Phase Change Materials“, Energy Conversion and Management, Vol. 95, pp. 69-89, 2015. $\uparrow 157$

[58] W. Liang Et Al., "Halloysite Clay Nanotubes Based Phase Change Material Composites with Excellent Thermal Stability for Energy Saving and Storage“, Rsc Advances, Vol. 6, No. 24, pp. 19669-19675, 2016. $\uparrow 157$

[59] Z. Ma, W. Lin, and M. I. Sohel, "Nano-Enhanced Phase Change Materials for Improved Building Performance", Renewable and Sustainable Energy Reviews, Vol. 58, pp. 1256- 1268, 2016. $\uparrow 157$

[60] K. Kaygusuz and A. Sari, "High Density Polyethylene/Paraffin Composites as Form- Stable Phase Change Material for Thermal Energy Storage“, Energy Sources, Part A: Recovery, Utilization and Environmental Effects, Vol. 29, No. 3, pp. 261-270, 2007. $\uparrow 157$

[61] A. Sarı, "Form-Stable Paraffin/High Density Polyethylene Composites as Solid-Liquid Phase Change Material for Thermal Energy Storage: Preparation and Thermal Properties", Energy Conversion and Management, Vol. 45, No. 13, pp. 2033-2042, 2004. $\uparrow 157$

[62] J. Li, P. Xue, W. Ding, J. Han, and G. Sun, "Micro-Encapsulated Paraffin/High-Density Polyethylene/Wood Flour Composite as Form-Stable Phase Change Material for Thermal Energy Storage“, Solar Energy Materials and Solar Cells, Vol. 93, No. 10, pp. 1761-1767, 2009. $\uparrow 158$

[63] C. Liu, L. Ma, Z. Rao, and Y. Li, "Synthesis and Thermal Properties of Magnesium Sulfate Heptahydrate/Urea Resin as Thermal Energy Storage Micro-Encapsulated Phase Change Material“, Journal of Heat Transfer, Vol. 140, No. 1, Art. No. 014501, pp. 231- 248, 2018. 1158

[64] Z. Qiu, X. Ma, P. Li, X. Zhao, and A. Wright, "Micro-Encapsulated Phase Change Material (Mpcm) Slurries: Characterization and Building Applications", Renewable and Sustainable Energy Reviews, Vol. 77, pp. 246-262, $2017 \uparrow 158$

[65] W. Su, J. Darkwa, and G. Kokogiannakis, "Review of Solid-Liquid Phase Change Materials and Their Encapsulation Technologies“, Renewable and Sustainable Energy Reviews, Vol. 48, pp. 373-391, 2015. $\uparrow 159$

[66] E. Oró, A. De Gracia, A. Castell, M. M. Farid, and L. F. Cabeza, "Review On Phase Change Materials (Pcms) for Cold Thermal Energy Storage Applications“, Applied Energy, Vol. 99, pp. 513-533, 2012. $\uparrow 159$

[67] P. Pardo, A. Deydier, Z. Anxionnaz-Minvielle, S. Rougé, M. Cabassud, and P. Cognet, "A On High Temperature Thermochemical Heat Energy Storage“, Renewable and Sustainable Energy Reviews, Vol. 32, pp. 591-610, 2014. $\uparrow 159$

[68] U. Pelay, L. Luo, Y. Fan, D. Stitou, and M. Rood, "Thermal Energy Storage Systems for Concentrated Solar Power Plants“, Renewable and Sustainable Energy Reviews, Vol. 79, pp. 82-100, 2017. $\uparrow 159$

[69] M. Bhouri and I. Bürger, "Numerical Investigation of H2 Absorption in An Adiabatic High-Temperature Metal Hydride Reactor Based On Thermochemical Heat Storage: $\mathrm{Mgh} 2$ and $\mathrm{Mg}(\mathrm{Oh}) 2$ as Reference Materials“, International Journal of Hydrogen Energy, Vol. 42, No. 26, pp. 16632-16644, 2017. $\uparrow 159$

[70] A. Jabbari-Hichri, S. Bennici, and A. Auroux, "Cacl2-Containing Composites as Thermochemical Heat Storage Materials“, Solar Energy Materials and Solar Cells, Vol. 172, pp. 177-185, 2017. $\uparrow 159$

[71] A. Krothapalli and B. Greska, "Concentrated Solar Thermal Power", In Handbook of Climate Change Mitigation and Adaptation, Second Edition, Vol. 2: Springer International Publishing, pp. 1503-1536, 2016. $\uparrow 160$

[72] L. Xie, L. Tian, L. Yang, Y. Lv, and Q. Li, "Review On Application of Phase Change Material in Water Tanks", Advances in Mechanical Engineering, Vol. 9, No. 7, pp. 203-214, 2017. $\uparrow 160$

[73] S. Kuravi, J. Trahan, D. Y. Goswami, M. M. Rahman, and E. K. Stefanakos, "Thermal Energy Storage Technologies and Systems for Concentrating Solar Power Plants“, Progress in Energy and Combustion Science, Vol. 39, No. 4, pp. 285-319, 2013. $\uparrow 160$

[74] A. Datas, A. Ramos, A. Martí, C. Del Cañizo, and A. Luque, "Ultra High Temperature Latent Heat Energy Storage and Thermophotovoltaic Energy Conversion“, Energy, Vol. 107, pp. 542-549, 2016. $\uparrow 160$ 


\section{Debrayan Bravo Hidalgo}

Ingeniero Mecánico, Universidad de Cienfuegos “Carlos Rafael Rodríguez”; magíster en Eficiencia Energética, Universidad de Cienfuegos; fue docente investigador, Centro de Estudios de Energía y Medio Ambiente (CEEMA), Universidad de Cienfuegos; editor de la Revista Publicando del grupo empresarial Research Management Learning (RML), Quito, Ecuador.

Correo electrónico: dbravo@rmlconsultores.com 